\title{
A Brief Study of Dietary Salt Intake in an Urban Population
}

\author{
Tahere Soltani ${ }^{1}$ iD) Seyed Saeed Mazloomy Mahmoodabad ${ }^{1 *}$ (iD, Azadeh Nadjarzadeh ${ }^{2}$ (D) , Ali Akbar Vaezi ${ }^{3}$ (D), \\ Seyedeh Mahdieh Namayandeh ${ }^{4}$ iD, Mohammad Hossein Soltani ${ }^{5}$ iD. Hossien Fallahzadeh ${ }^{6}$ iD \\ Received: 9 October 2020 Accepted: 20 December 2020 \\ Published 2021 Volume 2, Issue 1,
}

\begin{abstract}
Background: The purpose of this study is to investigate the amount of salt consumed by the people living in Yazd, Iran and its related factors based on previous investigations.

Methods: A general search was performed on electronic databases of Daneshgostar Barakat system, Magiran, SID and English databases such as Scopus, PubMed, Web of sciences, Science direct, and Google scholar search engines using following keywords: "salt intake" OR "sodium intake" OR "salt reduction" OR "salt content" OR "sodium reduction" OR "Diet, Reducing" regardless of the time interval. A total of 273 articles were obtained from the mentioned website. Papers published up to September 30, 2019 were retrieved. A total of 15 related articles were analyzed, and studies on the amount of salt consumed were evaluated.

Results: The amount of salt received by the people of Yazd is higher than the standard of the World Organization and has an increasing trend.

Conclusion: A population-based approach for salt intake reduction can lower blood pressure levels and presumably significantly reduce mortality. Therefore, the implementation of a comprehensive plan and intervention for salt consumption is necessary for this population.
\end{abstract}

Keywords: Intake salt, sodium intake, Yazd

@) How to Cite: Soltani T, Mazloomy Mahmoodabad SS, Nadjarzadeh A, Vaezi AA, Namayandeh SM, Soltani MH, et al. A Brief Study of Dietary Salt Intake in an Urban Yazd Population. Critical Comments in Biomedicine. 2021; 2(1): e10031.

$\square$ Seyed Saeed Mazloomy Mahmoodabad mazloomy@gmail.com

\footnotetext{
${ }^{1}$ Department of Health Education, Social Determinants of Health Research Center, School of Public Health, Shahid Sadoughi University of Medical Sciences, Yazd, Iran

${ }^{2}$ Department of Nutrition, Nutrition and Food Security Research Center, School of Public Health, Shahid Sadoughi University of Medical Sciences, Yazd, Iran

3 Department of Nursing, Research Center for Nursing \& Midwifery Care in Family Health, School of Nursing and Midwifery Care, Shahid Sadoughi University of Medical Sciences, Yazd, Iran

4 Afshar Research Development Center, Shahid Sadoughi University of Medical Sciences, Yazd, Iran

${ }^{5}$ Department of Cardiology, School of Medicine, Cardiovascular Research Center, Shahid Sadoughi University of Medical Sciences, Yazd, Iran

${ }^{6}$ Departments of Biostatistics and Epidemiology, Center For Healthcare Data Modeling, School of Public Health, Shahid Sadoughi University of Medical Sciences, Yazd, Iran
}

\section{Introduction}

Nowadays, changing lifestyle and consequently changing food consumption patterns and inadequate physical activity lead to non-communicable diseases such as high blood pressure, cardiovascular disease, diabetes, and various cancers as the most leading causes of death in the world [1]. Changes in dietary habits such as uncontrolled consumption of fast foods, salty snacks, carbonated beverages, sugary and sweet substances, low consumption of fruits and vegetables are the most important risk factors for non-communicable diseases; especially excessive salt consumption, is regarded as one of the main causes of these diseases [2].

In a case study on 2,000 people in Yazd, it was concluded that the prevalence of hypertension was $25.6 \%$ in those aged $20-74$ years in urban areas, that 
this prevalence is $23.3 \%$ in women and $27.5 \%$ in men [3].

There is some evidence that there is a link between salt intake and blood pressure as one of the main causes of cardiovascular disease [4]. Also, prospective studies and experimental results have shown that less salt intake is associated with reduced heart disease risk $[5,6]$.

If the amount of salt consumption in a community is reduced by 4.5 grams/day, systolic blood pressure in people with high blood pressure and healthy people will decrease by 4.8- and 1.9-mm $\mathrm{Hg}$, respectively[7] .Previous meta-analyses also showed that the reduction of salt by 2-3 grams per day leads to a $20-30 \%$ reduction in cardiovascular disease incidence [8-10]. According to the recommendation of the World Health Organization [11], the amount of salt intake from all food sources should be a maximum of 5 grams per day. The purpose of this study was to evaluate the amount of salt consumed by the people of Yazd, and the related factors based on other studies conducted in the city of Yazd.

\section{Materials and Methods}

This study is a review study that the results were obtained by searching the Iraian databases of Daneshgostar Barakat system, Magiran, SID and international databases such as Scopus PubMed, Web of sciences, Science direct and Google scholar search engine using following keywords: "salt intake" OR "sodium intake" OR "salt reduction" OR "salt content" OR "sodium reduction" OR "Diet, Reducing" regardless of the time interval.

A total of 273 articles were obtained from the mentioned website. Seven articles were deleted due to duplication. A total of 266 titles and abstracts were reviewed, and 30 of which were fully studied. Twelve articles were selected, and other articles were deleted due to irrelevance. Two cases were included in the study by reviewing the references, and a total of 15 related articles were reported (Figure. 1).

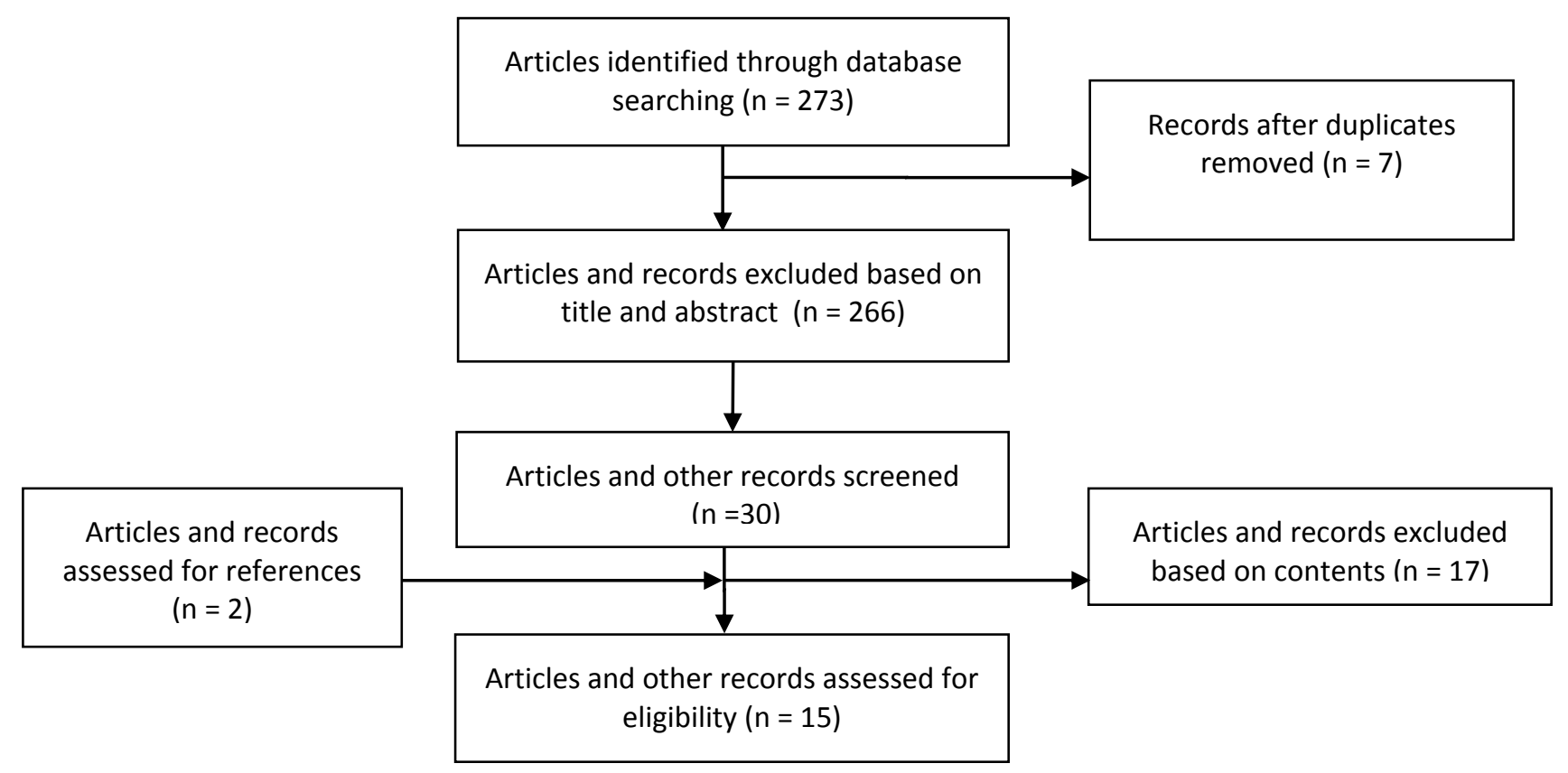

Figure 1: Diagram for the search and selection process of articles considered in this review 


\section{Results}

\section{1- Dietary salt intake}

The majority of studies ( $\mathrm{n}=5$ ) estimated sodium intake using mean 24-hour urinary sodium levels. Only one study was performed through Spot urine sampling. A study on the amount of salt intake of Yazd people in 2008 was done by Gharaghi and et al. with the aim of daily salt intake of adults in Yazd[12].In this study, salt intake through 24-hour urinary sodium excretion was estimated to be $9.13 \pm 4.5$. The average urinary sodium was $156.22 \pm$ $77.36 \mathrm{mEq} / 1$.

Motlagh et al. in 2011, estimated the average daily salt intake based on Kawasaki formula which was $10.09 \pm 2.97 \mathrm{~g}$ salt per day by studying the urine samples of 250 women referred to the health center. Daily salt intake of $4.1 \%$ (10 persons) of participants was less than 5g. [13]. Mirzaei et al by studying the urine samples of 300 urban populations aged20 to 74 years, reported on the average $9.2 \pm 3.5$ gr salt per day[14].

According to Akhondzadeh et al., the average salt intake in 100 patients with heart failure reported was estimated to be $8.04 \pm 3.01 \mathrm{~g} /$ day [15], and in a study done by Mirzaei et al. on 219 volunteers which evaluated daily salt intake through 24-hour urine, the mean daily dietary salt was $10 \pm 4.8 \mathrm{~g} /$ day in men and $7.5 \mathrm{~g} /$ day in women [14]. In the study done by Rahimdel et al., the average salt intake through 24hour urinary sodium excretion was $12.7 \mathrm{~g} /$ day [16].

Owing to the nature of the review, salt consumption has been increasing, unlike developed countries where sodium consumption is declining. Daily sodium consumption in Yazd city is much higher than world standards.

\section{Factors associated with high salt intake}

A study done in Yazdi people tried to measure the sodium content concluded that the mean salt content of traditional bread was significantly more than the standard level. Furthermore, Taftoon and Tanuri breads had significantly more salt compared with other breads [17]. On the other hand, studies showed that factors such as excess weight, dyslipidemia, and hypertension were the most common risk factors in Yazd region, and about $85 \%$ of Yazd citizens had at least one risk factor, and $61.1 \%$ had at least two coronary artery risk factors. A study showed that weight, dyslipidemia, and hypertension were the most prevalent risk factors in this region. A survey of 2,000 citizens showed that $42 \%$ of them had hypertension[3]. The relationship between eating habits and changes in metabolic syndrome components and other cardiovascular factors in a 10year study of the adult population showed that participants who ate fast food regularly had a higher waist circumference than others of the same age. This relationship was more prevalent and significant in women, so eating habits affect metabolic syndrome[18] .

Mazloomy et al. showed that the estimated salt intake was somewhat higher in subjects with a lower social background, while the opposite was true for lipid profile levels (LDL and HDL cholesterol) [19] .

Furthermore, in a study by Morowati et al., the reason for salt consumption was the misconception of participants about salt consumption, including hyperlipidemia and muscle contraction problems[20] . In the Motlagh study, the most important predictor of avoiding adding salt to food was attitude change[21] .

The majority of studies in this area were dedicated to the study of salt consumption and the prevalence of other risk factors affecting cardiovascular diseases. Considering the high prevalence and low awareness, treatment and control of hypertension and the prevalence of other risk factors, preventive community-based interventions in lifestyle, in particular, eating habits are highly needed.

\section{Conclusion}

In conclusion, a population-based approach can lead to reducing blood pressure levels and presumably a significant reduction in mortality and the starting of high blood pressure. In future research and public 
education initiatives, comprehensive implementation of a complete program and intervention is necessary for society to reduce salt consumption.

\section{Acknowledgments}

This study was extracted from a doctoral dissertation which was approved by the Research and Technology Deputy of Shahid Sadoughi University of Medical Sciences (ethical code: IR.SSU.SPH.REC.1397.141).

Authors' contribution

All authors contributed to this project and article equally. All authors read and approved the final manuscript.

Funding source

None

\section{Conflict of interest}

No competing financial or other interests exist.

\section{References}

[1] Nasehi MM, Moosazadeh M, Amiresmaeili M, Zakizadeh R, Mirzajani M. Prevalence of five main risk factors of non-communicable diseases in Mazandaran province: a population based study. Journal of Mazandaran University of Medical Sciences. 2012;21:193-202

[2] World Health Organization. Preventing chronic diseases. A vital investment: WHO global report Geneva. World Health Organization. 2005: 200:.

[3] Abadi AD-R, Mozaffari-Khosravi H, Nemayandeh $M$, Soltani MH, Mirzaei M, Abargouei AS. Sodium Status and Its Association with Overweight and Obesity in Adults Living in Yazd, Iran. Journal of Nutrition and Food Security. 2019

[4] Najafi-Ghezeljeh T, Akhondzadeh K. Adherence to sodium restriction and adherence to it in patients with heart failure: A review literature. Iranian Journal of Cardiovascular Nursing. 2016;4:56-63

[5] Abboud HD, Arnaout MS. Assessing and Improving the Knowledge Deficit about Salt Reduction: Effect of Salt Reduction Awareness on Lebanese Cardiac Subjects. Lebanese Medical Journal. 2016;103: 1-6

[6] He FJ, Burnier M, MacGregor GA. Nutrition in cardiovascular disease: salt in hypertension and heart failure. European heart journal. 2011;32:3073-80
[7] Nakagawa H, Miura K. Salt reduction in a population for the prevention of hypertension. Environmental health and preventive medicine. 2004;9:123-9

[8] He FJ, MacGregor GA. Reducing population salt intake worldwide: from evidence to implementation. Progress in cardiovascular diseases. 2010;52:363-82

[9] He FJ, MacGregor GA. Salt reduction lowers cardiovascular risk: meta-analysis of outcome trials. The Lancet. 2011;378:380-2

[10] Taylor RS, Ashton KE, Moxham T, Hooper L, Ebrahim S. Reduced dietary salt for the prevention of cardiovascular disease: a meta-analysis of randomized controlled trials (Cochrane review). American journal of hypertension. 2011;24:843-53

[11] World Health Organization. Reducing salt intake in populations: report of a WHO forum and technical meeting. meeting, 5-7 October 2006, Paris, France. 2007

[12] Gherahi Ghahi N. Evaluation of Daily Salt Intake in Adults in Yazd [MD Thesis] Yazd University of Medical Sciences. Iran. 2008

[13] Motlagh Z, Mazloomy S, MOZAFFARI KM MM, Askarshahi $H$. Salt intake among women refer to medical health centers. Yazd, Iran. 2011;2011

[14] Mirzaei $M$, Soltani $M$, Namayandeh $M$, GharahiGhehi N. Sodium and potassium intake of urban dwellers: nothing changed in Yazd, Iran. Journal of health, population, and nutrition. 2014;32: 111

[15] Akhondzadeh K, Ghezeljeh TN, Haghani H. The effect of the education program on the adherence intention to the dietary sodium restriction and the amount of sodium intake in patients with chronic heart failure. Iranian Red Crescent Medical Journal. 2018

[16] Rahimdel $T$, Morowatisharifabad $M$, SalehiAbargouei A, Mirzaei $M$, Fallahzadeh $H$. Evaluation of an education program based on the theory of planned behavior for salt intake in individuals at risk of hypertension. Health education research. 2019

[17] Namayandeh SM, Lotfi MH, Jafari V, Dad V, Biabani J, Razi $\mathrm{MH}$, et al. Salt Content in Traditional and Nontraditional Breads in Yazd City, Iran, 2015-2016. Journal of Nutrition and Food Security. 2018;3:185-92

[18] Sarebanhassanabadi M, Mirhosseini SJ, Mirzaei M, Namayandeh SM, Soltani $\mathrm{MH}$, Pakseresht $M$, et al. Effect of dietary habits on the risk of metabolic syndrome: Yazd Healthy Heart Project. Public health nutrition. 2018;21:1139-46

[19] Mazloomy Mahmoodabad SS, Tehrani H, Gholianaval $M$, Gholami $H$, Nematy $M$. The effect of social class on the amount of salt intake in patients with hypertension. Blood pressure. 2016;25:360-3 
[20] Morowatisharifabad MA, Salehi-Abargouei A, Mirzaei $M$, Rahimdel T. Behavioral beliefs of reducing salt intake from the perspective of people at risk of hypertension: An exploratory study. ARYA Atherosclerosis. 2019;15

[21] Motlagh Z, Mazloomy S, Morowaty M.
Determinants of salt consumption based on theory of planned behavior among women refered to settings of health care, Yazd, Iran, 2010. The First International \& 4th National Congress on health Education \& Promotion, 2011: Tabtiz university of medical sciences; 2011 